\title{
A educação alimentar e nutricional nas escolas no combate à fome e a desnutrição infantil: uma revisão bibliográfica
}

\author{
Food and nutrition education in schools to combat hunger and child malnutrition: a \\ bibliographic review
}
Educación alimentaria y nutricional en las escuelas para combatir el hambre y la desnutrición infantil: una revisión bibliográfica

\begin{abstract}
Ronneo Lúcio Silva Rodrigues ${ }^{1 *}$, Rubens da Silva Carvalho ${ }^{1}$, Elivania de Souza Benevides ${ }^{1}$, Dalvina Costa Fontana ${ }^{1}$, Paulina Pedro das Neves ${ }^{1}$, Alícia Real Tuão ${ }^{1}$, Evilásio Mussy Caetano Junior ${ }^{1}$, Dilmeia Fernandes Pacheco ${ }^{1}$, Delcenir Porto Costalonga1, Valkiria Santana das Neves Cruz ${ }^{1}$.
\end{abstract}

\section{RESUMO}

Objetivo: Apresentar como a educação alimentar e nutricional nas escolas promove o combate à fome e a desnutrição infantil. Revisão bibliográfica: A fome e a desnutrição infantil foram problemas recorrentes no Brasil nas décadas passadas, e com a crise econômica vem se tornando um novo problema social que dificilmente será sanado a curto prazo. Instruir através do ambiente escolar políticas alimentares saudáveis e sustentáveis se faz uma maneira de lidar com o problema, construindo uma ponte entre educação e saúde pública, na busca por uma sociedade mais saudável e menos afetada pela desigualdade social. A escola é o ambiente que garante a alimentação de muitas crianças e adolescentes no Brasil, fazendo com que a baixa renda de muitas famílias não favoreça a falta de insumos básicos. Uma ou duas alimentações diárias no cotidiano escolar representa uma alternativa para a redução dos dados da desnutrição, e abre portas para que o Estado poça inserir alimentações mais saudáveis e nutritivas. Considerações finais: Compreende-se, portanto, que o direito à vida está completamente ligado às garantias educacionais e alimentares, e que a escola é um ambiente propício para promover o contato das futuras gerações com a boa alimentação.

Palavras-chave: Assistência alimentar, Nutrição, Educação alimentar e nutricional.

\begin{abstract}
Objective: To present how food and nutrition education in schools promotes the fight against child hunger and malnutrition. Bibliographic review: Hunger and child malnutrition have been recurrent problems in Brazil in the past decades, and with the economic crisis it has become a new social problem that will hardly be solved in the short term. Educating healthy and sustainable food policies through the school environment is a way of dealing with the problem, building a bridge between education and public health, in the search for a healthier society and less affected by social inequality. The school is the environment that guarantees the nutrition of many children and adolescents in Brazil, making sure that the low income of many families does not favor the lack of basic inputs. One or two daily meals in school life represents an alternative for reducing malnutrition data and opens doors for the State to insert healthier and more nutritious food. Final considerations: It is understood, therefore, that the right to life is completely linked to educational and food guarantees, and that the school is a favorable environment to promote the contact of future generations with good food.
\end{abstract}

Key words: Food assistance, Nutrition, Food and nutrition education.

\footnotetext{
${ }^{1}$ Faculdade Vale do Cricaré (FVC), Espírito Santo - BR. *E-mail: ronneolucio@hotmail.com

2 Faculdade Mineira (UNIMINAS), Juiz de Fora - MG.
} 


\section{RESUMEN}

Objetivo: Presentar cómo la educación alimentaria y nutricional en las escuelas promueve la lucha contra el hambre y la desnutrición infantil. Revisión bibliográfica: El hambre y la desnutrición infantil han sido problemas recurrentes en Brasil en las últimas décadas, y con la crisis económica se ha convertido en un nuevo problema social que difícilmente se resolverá en el corto plazo. Educar políticas alimentarias saludables y sostenibles a través del entorno escolar es una forma de abordar el problema, construyendo un puente entre la educación y la salud pública, en la búsqueda de una sociedad más saludable y menos afectada por la desigualdad social. La escuela es el entorno que garantiza la nutrición de muchos niños y adolescentes en Brasil, asegurándose de que los bajos ingresos de muchas familias no favorezcan la falta de insumos básicos. Una o dos comidas diarias en la vida escolar representa una alternativa para reducir los datos de desnutrición, y abre puertas para que el Estado inserte alimentos más saludables y nutritivos. Consideraciones finales: Se entiende, por tanto, que el derecho a la vida está completamente ligado a las garantías educativas y alimentarias, y que la escuela es un entorno propicio para promover el contacto de las generaciones futuras con la buena alimentación.

Palabras clave: Asistencia alimentaria, Nutrición, Educación alimentaria y nutricional.

\section{INTRODUÇÃO}

A Educação Alimentar e Nutricional (EAN) é conceituada como um objeto de ação interdisciplinar e interdepartamental, em que o conhecimento e a aprendizagem contínuas visam promover continuamente hábitos alimentares saudáveis, desenvolver autonomia e voluntariedade, e recursos educacionais ativos e problemáticos. Portanto, a educação alimentar e nutricional desempenha um papel importante na promoção de hábitos alimentares saudáveis e é uma estratégia indispensável nas políticas públicas de alimentação e nutrição (FRANÇA CJ e CARVALHO VCHS, 2017).

Com o tempo, a educação nutricional passou por mudanças importantes. Percebe-se que, na década de 1940, a estratégia baseava-se no ensino da alimentação dita correta, com aparência puramente biológica e baseada no binômio educação alimentar. Desde a década de 1970, as políticas de alimentação e nutrição foram alteradas, nas quais a renda é vista como a principal dificuldade para a obtenção de alimentos saudáveis (FRANÇA CJ e CARVALHO VCHS, 2017).

É importante notar também que entre 1990 e 2010, os indivíduos foram inicialmente considerados como detentores de direitos e, em seguida, solicitados a expandir seu poder de escolha e decisão. A Estratégia Fome Zero, lançada em 2001, também considerou uma abordagem semelhante, considerando a importância da educação alimentar relacionada à educação do cidadão, o que em matéria de educação alimentar parece ter um significado estratégico em seu campo. O mesmo pode ser verificado na Política Nacional de Segurança Alimentar e Nutricional aprovada em 2010, que visa garantir o direito à alimentação adequada com base em práticas alimentares saudáveis (RAMOS FP, et al., 2013).

Dentre os itens da agenda pública existentes para o alívio das consequências da fome, destaca-se o Programa Nacional de Alimentação Escolar (PNAE), onde a alimentação escolar brasileira não só prevê a erradicação da fome, mas também oferece educação alimentar e nutricional e satisfação. As necessidades nutricionais dos alunos permitiram promover o crescimento e o desenvolvimento biológico psicossocial, a aprendizagem, o rendimento escolar e a formação de hábitos alimentares saudáveis. O abastecimento, acesso e qualidade dos alimentos é uma preocupação urgente. O Brasil demorou 25 anos (de 1990 a 2014) para reduzir a proporção de pessoas extremamente pobres, saindo do Mapa da Fome da Organização das Nações Unidas para a Alimentação e Agricultura (FAO). Mas em 2018, já havia 13 milhões de pessoas extremamente pobres (6,5\% da população) enfrentando restrições de acesso aos alimentos (BICALHO D e LIMA TM, 2020).

O PNAE tem por objetivo avaliar o estado nutricional de crianças como ferramenta importante de verificar a saúde da comunidade escolar. A identificação de crianças com distúrbios nutricionais pode ajudar a nortear 
medidas de promoção e intervenção em saúde, não só na infância, mas também na juventude e na idade adulta para redução de doenças. A escola é um espaço único para o desenvolvimento dessas ações, pois se constitui em um espaço privilegiado para a construção de valores e compreensão do mundo, interferindo na produção social saudável. No Brasil, pela natureza intersetorial do sistema único de saúde (SUS), a escola é um ambiente político e praticamente estreito entre os setores de saúde e educação através do Programa Nacional de Alimentação Escolar (PNAE) e Programa de Saúde na Escola (PSE), que visam promover alimentação saudável, saúde e nutrição na rede pública de ensino (LOURENÇO AEP, et al., 2019).

De acordo com Yokota RTC, et al. (2010), o ponto de partida para a promoção da alimentação saudável no ambiente escolar é a partir de uma perspectiva holística e multidisciplinar das pessoas, que leva em consideração os seres humanos, especialmente a família, a comunidade e o contexto social da escola. Também busca desenvolver conhecimentos, destrezas e habilidades em todas as oportunidades educacionais para o autocuidado e prevenção de comportamentos perigosos.

Além disso, facilita a análise crítica e reflexiva de valores, comportamentos, condições sociais e estilos de vida e se empenha em fortalecer tudo o que contribui para a melhoria da saúde e do desenvolvimento humano. Com base nisso, as agências internacionais sempre recomendaram fortemente a promoção da saúde no ambiente escolar e este trabalho é dirigido principalmente a crianças com mais de cinco anos que, embora sejam vulneráveis aos aspectos biológicos, nutricionais e sociais, ainda não estão inseridas no enfoque estratégico da política de saúde em geral (YOKOTA RTC, et al., 2010).

Elucidados pela literatura existente na introdução desta revisão, o objetivo dessa revisão é reunir a literatura existente para apresentar como a educação alimentar e nutricional nas escolas promove o combate à fome e a desnutrição infantil.

\section{REVISÃO BIBLIOGRÁFICA}

A alimentação adequada é um direito humano básico. Uma das recomendações do governo para garantir esse direito é permitir que grupos vulneráveis recebam programas de transferência de renda e instrução escolar sobre boa alimentação. O objetivo geralmente é melhorar suas condições de vida. Entre outras coisas, outros suprimentos são adicionados que fomentem o a cesso à alimentação (DE PAULA DV, et al., 2012).

"A emenda constitucional 64 dispõe que: Art. 1ํO art. 6ำ da Constituição Federal passa a vigorar com a seguinte redação: Art. 6 São direitos sociais a educação, a saúde, a alimentação, o trabalho, a moradia, o lazer, a segurança, a previdência social, a proteção à maternidade e à infância, a assistência aos desamparados, na forma desta Constituição" (BRASIL, 2010).

O crescimento físico é o parâmetro mais adequado para avaliar a saúde e o estado nutricional das crianças, pois reflete o desenvolvimento saudável e populacional. Portanto, o diagnóstico de problemas nutricionais e o desenvolvimento de pesquisas de conhecimento tornam-se significativos. A desnutrição é a causa de lesões graves na infância, sendo responsável por mais de um terço de todas as mortes infantis em todo o mundo. Esta é uma questão preocupante porque cerca de 178 milhões de crianças no mundo são de baixa estatura, refletindo a existência de subnutrição e doenças (PEDRAZA DF, et al., 2017).

Tal realidade só pode ser revertida com mecanismos eficazes, como por exemplo, utilizando-se da estrutura do Estado para alcançar o cerne do problema. O ambiente escolar é considerado um espaço estratégico para as ações de educação alimentar por incluir uma estrutura pedagógica organizada. Os alunos passam muito tempo na escola, oferecendo uma ou duas refeições por dia, cinco dias por semana (DE PAULA DV, et al., 2012).

Há também o fato de o aluno representar potenciais agentes de mudança em sua família e comunidade. Casa e escola têm uma grande influência no ambiente em que os hábitos alimentares das crianças são formados. Isso porque as crianças aprendem a compreender as características dos alimentos não apenas por meio da experiência, mas também por meio da observação e imitação de pais, professores e colegas. No 
entanto, poucos são os estudos que descrevem a qualidade da alimentação e as características antropométricas de crianças de famílias com e sem pensão (DE PAULA DV, et al., 2012).

No ambiente escolar, também faltam referenciais teóricos e metodológicos para embasar a prática da EAN. Pode-se entender que a formação dos hábitos alimentares é afetada por fatores físicos, psicológicos, sociais, culturais e econômicos que começaram a se formar na infância. Nesse caso, os hábitos alimentares são considerados parte integrante dos hábitos alimentares e, com o tempo, os hábitos alimentares continuarão a se repetir, sendo importante ressaltar que o indivíduo se afasta nesta fase da vida (RAMOS FP, et al., 2013).

Ainda para Ramos FP, et al. (2013), familiarizado basicamente com o ambiente e entrar no ambiente escolar, experimentará outros alimentos e preparações, e devido à influência de grupos sociais e fatores estimuladores existentes no sistema de ensino, terá a oportunidade de promover mudanças nos hábitos alimentares. Portanto, é compreensível que esta escola proporcione um ambiente de excelência para o programa EAN e tenha levado em consideração esse momento crítico na formulação das políticas públicas de alimentação e nutrição

A alimentação que a maioria dos pais ou responsáveis dá aos filhos é semelhante à da infância e da adolescência, como os alimentos industrializados ricos em açúcares e gorduras, muito comuns há pouco tempo. Muitos desses alimentos prejudicam a saúde geral do indivíduo, tornando-o sedentário e altamente nutrido na idade adulta. Porém, na realidade, a alimentação é uma atividade básica contínua no contexto do desenvolvimento e das relações sociais. Parte dessa alimentação é feita em grupos e espaços sociais. Portanto, neste sentido, a escola superou o conceito simples de lugar de aprendizagem e passou a ser um lugar de alimentação e cooperação (SANTOS KS, et al., 2020).

Ao se utilizar as escolas e principalmente o PNAE como locais de educação alimentar e nutricional, é necessário definir quais profissionais inserir nesses processos educativos. Em estudo com alunos de cursos ministrados por nutricionistas e professores, concluiu-se que os alunos que frequentaram aulas com nutricionistas tiveram desempenho inferior nas disciplinas abordadas. O mesmo autor destacou que esse resultado pode ser explicado pelo maior contato entre professores e alunos, comunicação descontraída e inserção da realidade social e cultural dos alunos (FERNANDES AGS, et al., 2014).

\section{O Nutricionista frente ao Programa Nacional de Alimentação Escolar (PNAE)}

O nutricionista é um clínico geral com a responsabilidade de promover a segurança alimentar e os cuidados nutricionais em todas as áreas de atividade, ajudando a manter e restaurar o estado nutricional de indivíduos e comunidades. Com a regulamentação da profissão em 1967, houve um aumento significativo dos cursos de graduação no Brasil e, consequentemente, do número de especialistas nesta área, o que levou ao desenvolvimento de várias áreas de atuação desse especialista (SOUZA AA, et al., 2017).

O perfil de saúde da população brasileira e a mudança epidemiológica em curso no Brasil, em decorrência das mudanças no perfil demográfico e nutricional da população, têm promovido profundas mudanças nos padrões de morbimortalidade e no estado nutricional de todas as faixas etárias da área geográfica, em áreas urbanas e rurais de grandes e pequenos municípios. Deve-se destacar que em nosso país existem doenças típicas de países subdesenvolvidos, causadas pela fome e desnutrição, decorrentes da má alimentação e subnutrição, além de um estilo de vida inadequado. É neste contexto que se justifica a inclusão do nutricionista em inúmeros campos da sociedade (GEUS LMM, et al., 2011).

De acordo com Souza AA, et al. (2017), no "Programa Nacional de Alimentação Escolar (PNAE)", os nutricionistas preconizam hábitos alimentares saudáveis na perspectiva de estabelecer a Segurança Alimentar e Nutricional (SAN) e respeitar a cultura alimentar local, que tem recebido cada vez mais atenção. Avanços significativos foram obtidos nas mudanças na estrutura jurídica do programa, incluindo o aumento da contratação de nutricionistas, o que possibilitou ao profissional tornar-se o Responsável técnico (RT) das atividades de alimentação escolar. As resoluções do Conselho Federal de Nutricionistas (CFN), editadas desde 2005, orientam e regulamentam as responsabilidades dos nutricionistas que trabalham no PNAE. De acordo com a Lei no 11.947/2009, o plano define nutricionista como profissional de RT, que deve estar 
vinculado ao departamento de alimentação da entidade fiscalizadora e cadastrado como RT na Fundação Nacional de Desenvolvimento Educacional (FNDE).

Nesse sentido, o PNAE enfatiza o papel do nutricionista que, embora possa começar no ambiente escolar, pode ajudar a mudar os hábitos alimentares da escola na perspectiva de uma alimentação saudável e buscando a segurança alimentar e nutricional. Nas últimas duas décadas, o programa obteve avanços significativos, como mudanças nos procedimentos legais e aumento da contratação de nutricionistas, consolidando a posição do profissional como técnico responsável pela alimentação escolar. Entre outras funções, ele também é responsável por coordenar o diagnóstico e o acompanhamento do estado nutricional dos alunos, planejando a merenda escolar com base na cultura alimentar, no perfil epidemiológico das pessoas atendidas e nas ocupações agrícolas da região. Da compra de alimentos à produção de alimentos e distribuição, além de propor e realizar ações de educação alimentar e nutricional nas escolas (MELLO AL, et al., 2012).

"A lei 11.947/2009 define o nutricionista da seguinte forma: Art. 11. A responsabilidade técnica pela alimentação escolar nos Estados, no Distrito Federal, nos Municípios e nas escolas federais caberá ao nutricionista responsável, que deverá respeitar as diretrizes previstas nesta Lei e na legislação pertinente, no que couber, dentro das suas atribuições específicas. Art. 12. Os cardápios da alimentação escolar deverão ser elaborados pelo nutricionista responsável com utilização de gêneros alimentícios básicos, respeitando-se as referências nutricionais, os hábitos alimentares, a cultura e a tradição alimentar da localidade, pautando-se na sustentabilidade e diversificação agrícola da região, na alimentação saudável e adequada. § 1ํ Para efeito desta Lei, gêneros alimentícios básicos são aqueles indispensáveis à promoção de uma alimentação saudável, observada a regulamentação aplicável. (Renumerado do parágrafo único Incluído pela Lei $n^{\circ}$ 12.982, de 2014) § 20 Para os alunos que necessitem de atenção nutricional individualizada em virtude de estado ou de condição de saúde específica, será elaborado cardápio especial com base em recomendações médicas e nutricionais, avaliação nutricional e demandas nutricionais diferenciadas, conforme regulamento. (Incluído pela Lei no 12.982, de 2014)

Art. 13. A aquisição dos gêneros alimentícios, no âmbito do PNAE, deverá obedecer ao cardápio planejado pelo nutricionista e será realizada, sempre que possível, no mesmo ente federativo em que se localizam as escolas, observando-se as diretrizes de que trata $o$ art. 20 desta Lei" (BRASIL, 2009).

Como forma de fiscalizar as resoluções acima mencionadas, desde 2005, a coordenação geral do PNAE/FNDE aprovou o formulário que deve ser encaminhado à coordenação, e os profissionais que atuam neste plano foram cadastrados de forma contínua e sistemática, devendo o nutricionista estar devidamente incluído e formalizados como representantes técnicos e incluídos nos Quadros Técnicos (QT) desses profissionais e seus gestores, para que o levantamento anual (FNDE) de dados cadastrais por meio do sistema de cadastro do nutricionista (SINUTRI) seja comparado com a regulamentação vigente. Dessa forma pode-se atualizar e descobrir possíveis inconsistências, além de acompanhar e interagir com nutricionistas que atuam na alimentação escolar para buscar aprimorar a implantação conjunta do PNAE. O SINUTRI também pode enviar relatórios ao CFN para que o desempenho dos profissionais seja monitorado e supervisionado (CHAVES LG, et al., 2013).

\section{A alimentação na escola fortalecendo políticas públicas de combate à desnutrição}

A desnutrição é responsável por mais de um terço das mortes infantis em todo o mundo. Estima-se que existam 178 milhões de crianças no planeta com escassez devido à dieta insuficiente, deficiência de vitaminas e minerais e à existência de doenças. É uma síndrome multifatorial caracterizada por crescimento linear prejudicado e/ou perda acentuada de peso em crianças. Está relacionada à incidência e gravidade de doenças infecciosas, aumento da mortalidade infantil, atrasos no desenvolvimento psicomotor, dificuldades no 
desempenho escolar e diminuição da produtividade na idade adulta. A principal causa da desnutrição costuma estar relacionada à pobreza, que leva à escassez de alimentos e a falta de boa alimentação, por isso é necessário compreender seus principais determinantes como renda, educação, saneamento, moradia e acesso à serviços de saúde (OLIVEIRA FCC, et al., 2011).

Uma estratégia do Estado para combater a desnutrição infantil é tornar o ambiente educacional um meio de acesso as crianças, e através da merenda escolar garantir outras alimentações aos alunos. $O$ termo "merenda escolar" tem sido utilizado para designar a merenda do Programa Nacional de Alimentação Escolar (PNAE). Portanto, nas escolas públicas de ensino fundamental e médio do Brasil, a alimentação escolar é um hábito alimentar relacionado. O comportamento alimentar na escola ocorre em outras práticas que compõem o espaço escolar, vivências e processos que dependem das vivências e processos que afetam os hábitos alimentares e a identidade de crianças e adolescentes (SILVA EO, et al., 2018).

Crianças e jovens de todo o mundo podem se beneficiar da merenda escolar. Fornecer alimentos saudáveis nas escolas pode não apenas ajudar a reduzir a taxa de abandono escolar, mas também apoiar o crescimento das crianças, fornecendo nutrição adequada e melhorando as habilidades cognitivas dos alunos. Além disso, em alguns países, os programas de alimentação escolar fazem parte do sistema de proteção social e, por meio da cooperação com comunidades vulneráveis para o uso de alimentos produzidos na agricultura local, o desenvolvimento local sustentável se tornou uma prioridade. Desta forma, estes programas operam na perspectiva da promoção de todos os aspectos da segurança alimentar e nutricional (SAN), nomeadamente a disponibilidade, acesso e utilização de alimentos e nutrição (CESAR JT, et al., 2018).

Além dos serviços de alimentos e alimentação, o meio ambiente e o ambiente alimentar também afetam as escolhas alimentares. O comportamento alimentar é complexo, incluindo determinantes internos e externos ao sujeito, considera os hábitos alimentares relacionados às escolhas alimentares, seu preparo e consumo propriamente dito, além de outros valores simbólicos. O comportamento alimentar é cheio de significado além do fato de satisfazer a fome e as necessidades nutricionais, e a fome e as necessidades nutricionais estão frequentemente relacionadas à dieta. É preciso compreender que as escolhas alimentares não estão relacionadas apenas a aspectos objetivos, como quantidade e qualidade dos alimentos, renda, mas também a aspectos subjetivos, como sabor, prazer, valor e relações sociais (ASSAO TY, et al., 2014).

Além disso, a merenda escolar também desempenha um papel importante na sociedade, pois em muitos casos, a merenda escolar é considerada a principal refeição do dia e a única garantia de alimentação das crianças. Diante desse quadro, é importante avaliar a adequação nutricional dos cardápios fornecidos pelas escolas brasileiras para verificar se atendem aos parâmetros propostos pelo PNAE. Essas informações nos permitirão propor intervenções para melhorar a qualidade da alimentação escolar, determinar que os alunos adotem hábitos alimentares saudáveis e reduzir os distúrbios nutricionais (ISSA RC, et al., 2014).

Embora existam normas e regulamentos para orientar o PNAE do país, algumas deficiências foram encontradas na gestão e implementação, tais como: oferta anormal, infraestrutura escolar insuficiente, falta de treinamento para chefes de cozinha, falta ou número insuficiente de nutricionistas e valores nutricionais baixos na comida fornecida. Portanto, avaliar e identificar as deficiências existentes é importante para 0 investimento em melhorias e manutenção. Isso ajudará os gestores na tomada de decisões e na compreensão da implementação de todas as etapas do PNAE. Embora o PNAE tenha uma longa história, poucas são as publicações sobre sua abrangência e relevância. A avaliação do plano público é fundamental para orientar o planejamento e a execução das ações. É importante encontrar situações anormais e experiências positivas para melhor reformular e planejar as atividades desse plano (ROCHA NP, et al., 2018).

O PNAE é custeado pelo Ministério da Fazenda e os recursos são garantidos pelo orçamento federal, por isso o Fundo Nacional de Desenvolvimento da Educação (FNDE) repassa seus recursos aos órgãos executores (estados, distritos federais e municípios) sem acordos formais, reajustes, acordos, contratos ou qualquer outra ferramenta. Portanto, a entidade executora deve ser incluída no censo escolar realizado pelo Instituto Nacional de Pesquisas Educacionais (Anísio Teixeira) (INEP) do Ministério da Educação, que quantifica o número total de alunos matriculados em cada unidade escolar. Além disso, as escolas filantrópicas também devem se cadastrar no Conselho Nacional de Assistência Social (CNAS) e declarar seu 
interesse em fornecer alimentação escolar com recursos federais aos alunos inscritos (DOS SANTOS SR, et al., 2016).

\section{CONSIDERAÇÕES FINAIS}

A educação alimentar e nutricional, além de ampliar a alimentação diária saudável de crianças e adolescentes matriculados na educação básica, fornecem importante auxílio nas políticas de combate a fome e desnutrição, propiciando uma oportunidade ao poder público de inserir alimentações saudáveis nos cardápios dos alunos, o que influencia diretamente na capacidade do aprendizado e no desenvolvimento corporal. A educação alimentar e nutricional não é apenas uma didática a ser desenvolvida nas salas de aula, mas uma forma de vida a ser incentivada entre os alunos e prosperada na sociedade que os cerca.

\section{REFERÊNCIAS}

1. ASSAO TY, et al. Alimentação escolar: o que o desenho infantil revela. Journal of Human Growth and Development, 2014; 24(1): 98-105.

2. BICALHO D, LIMA TM. O Programa Nacional de Alimentação Escolar como garantia do direito à alimentação no período da pandemia da COVID-19. Demetra, 2020; 1(15): 1-9.

3. BRASIL. Emenda Constitucional n 64, de 4 de Fevereiro de 2010, altera o art. 6ำ da Constituição Federal, para introduzir a alimentação como direito social. $2010 . \quad$ Disponível em: http://www.planalto.gov.br/ccivil_03/constituicao/emendas/emc/emc64.htm. Acesso em: 27 de dez. 2020.

4. BRASIL. Lei no 11.947, de 16 de Junho de 2009, Dispõe sobre o atendimento da alimentação escolar e do Programa Dinheiro Direto na Escola aos alunos da educação básica; altera as Leis nos 10.880, de 9 de junho de 2004, 11.273, de 6 de fevereiro de 2006, 11.507, de 20 de julho de 2007; revoga dispositivos da Medida Provisória no 2.178-36, de 24 de agosto de 2001, e a Lei no 8.913, de 12 de julho de 1994; e dá outras providências. 2009. Disponível em: http://www.planalto.gov.br/ccivil_03/_ato2007-2010/2009/lei/l11947.htm. Acesso em: 26 de dez. 2020.

5. CESAR JT, et al. Alimentação Escolār no Brasil e Estados Unidos: uma revisão integrativa. Ciência \& Saúde Coletiva, 2018; 23(3): 991-1007.

6. CHAVES LG, et al. Reflexões sobre a atuação do nutricionista no Programa Nacional de Alimentação Escolar no Brasil. Ciência \& Saúde Coletiva, 2013; 18(4): 917-926.

7. DE PAULA DV, et al. Avaliação nutricional e padrão de consumo alimentar entre crianças beneficiárias e não beneficiárias de programas de transferência de renda, em escola municipal do Município de Belo Horizonte, Estado de Minas Gerais, Brasil, em 2009. Epidemiologia e Serviços de Saúde, 2012; 21(3): 385-394.

8. DOS SANTOS SR, et al. As formas de gestão do programa nacional de alimentação escolar (PNAE). Revista de Salud Pública, 2016; 18(2): 311-320.

9. FERNANDES AGS, et al. Alimentação escolar como espaço para educação em saúde: percepção das merendeiras do município do Rio de Janeiro, Brasil. Ciência \& Saúde Coletiva, 2014; 19(1): 39-48.

10. FRANÇA CJ, CARVALHO VCHS. Estratégias de educação alimentar e nutricional na Atenção Primária à Saúde: uma revisão de literatura. Saúde Debate, 2017; 41(114): 932-948.

11. GEUS LMM, et al. A importância na inserção do nutricionista na Estratégia Saúde da Família. Ciência \& Saúde Coletiva, 2011; 16(1): 797-804.

12. ISSA RC, et al. Alimentação escolar: planejamento, produção, distribuição e adequação. Rev Panam Salud Publica, 2014; 35(2): 96-106.

13. LOURENÇO AEP, et al. influência da ambiência escolar no estado nutricional de pré-escolares de Macaé, rio de Janeiro, Brasil. Ciência \& Saúde Coletiva, 2019; 24(7): 2399-2410.

14. MELLO AL, et al. Perfil do nutricionista do programa nacional de alimentação escolar na região Nordeste do Brasil. Revista de Nutrição, 2012; 25(1): 119-132.

15. OLIVEIRA FCC, et al. Estado nutricional e fatores determinantes do déficit estatural em crianças cadastradas no Programa Bolsa Família. Epidemiol. Serv. Saúde, 2011; 20(1): 7-18.

16. PEDRAZA DF, et al. Estado nutricional e hábitos alimentares de escolares de Campina Grande, Paraíba, Brasil. Ciência \& Saúde Coletiva, 2017; 22(2): 469-477.

17. RAMOS FP, et al. Educação alimentar e nutricional em escolares: uma revisão de literatura. Cad. Saúde Pública, $2013 ; 29(11): 2147-2161$.

18. ROCHA NP, et al. Análise do programa nacional de alimentação escolar no município de Viçosa, MG, Brasil. Revista de Saúde Pública, 2018; 52(1): 1-19.

19. SANTOS KS, et al. O reflexo da Educação Alimentar e nutricional escolar nas condutas alimentares dos alunos e seu impacto na saúde geral: Uma abordagem conjunta. Revista Científica Multidisciplinar Núcleo do Conhecimento, 2020; 5(13): 44-56.

20. SILVA EO, et al. Alimentação escolar e constituição de identidades dos escolares: da merenda para pobres ao direito à alimentação. Cadernos de Saúde Pública, 2018; 34(4): 1-13.

21. SOUZA AA, et al. Atuação de nutricionistas responsáveis técnicos pela alimentação escolar de municípios de Minas Gerais e Espírito Santo. Ciência \& Saúde Coletiva, 2017; 22(2):593-606.

22. YOKOTA RTC, et al. Projeto "a escola promovendo hábitos alimentares saudáveis": comparação de duas estratégias de educação nutricional no Distrito Federal, Brasil. Revista de Nutrição, 2010; 23(1): 37-47. 